Article

\title{
Migraine Headaches after Major Surgery with General or Neuraxial Anesthesia: A Nationwide Propensity-Score Matched Study
}

\author{
Chung-Yi Liao ${ }^{1,2} \mathbb{D}$, Chun-Cheng Li ${ }^{1,2}$, Hsin-Yi Liu ${ }^{1,2}$, Jui-Tai Chen ${ }^{1,2}$, Yih-Giun Cherng ${ }^{1,2} \mathbb{D}$, \\ Tzeng-Ji Chen ${ }^{3,4} \mathbb{D}^{D}$, Ying-Xiu Dai ${ }^{4,5}{ }^{\mathbb{D}}$, Hsiang-Ling Wu ${ }^{4,6}$, Wan-Chi Liu ${ }^{1,2, *}$ and Ying-Hsuan Tai ${ }^{1,2, *(\mathbb{D})}$
}

1 Department of Anesthesiology, Shuang Ho Hospital, Taipei Medical University, New Taipei City 23561, Taiwan; 19206@s.tmu.edu.tw (C.-Y.L.); 15193@s.tmu.edu.tw (C.-C.L.); 18384@s.tmu.edu.tw (H.-Y.L.); $19240 @$ s.tmu.edu.tw (J.-T.C.); stainless@s.tmu.edu.tw (Y.-G.C.)

2 Department of Anesthesiology, School of Medicine, College of Medicine, Taipei Medical University, Taipei 11031, Taiwan

3 Department of Family Medicine, Taipei Veterans General Hospital, Taipei 11217, Taiwan; tjchen@vghtpe.gov.tw

4 School of Medicine, National Yang Ming Chiao Tung University, Taipei 11221, Taiwan; daiinxiu@gmail.com (Y.-X.D.); hlwu9@vghtpe.gov.tw (H.-L.W.)

5 Department of Dermatology, Taipei Veterans General Hospital, Taipei 11217, Taiwan

6 Department of Anesthesiology, Taipei Veterans General Hospital, Taipei 11217, Taiwan

* Correspondence: 16057@s.tmu.edu.tw (W.-C.L.); 18045@s.tmu.edu.tw (Y.-H.T.)

Citation: Liao, C.-Y.; Li, C.-C.; Liu, H.-Y.; Chen, J.-T.; Cherng, Y.-G.;

Chen, T.-J.; Dai, Y.-X.; Wu, H.-L.; Liu, W.-C.; Tai, Y.-H. Migraine Headaches after Major Surgery with General or Neuraxial Anesthesia: A Nationwide Propensity-Score Matched Study. Int . J. Environ. Res. Public Health 2022, 19, 362. https://doi.org/10.3390/ ijerph19010362

Academic Editors: Paul B. Tchounwou and Jimmy T. Efird

Received: 16 November 2021 Accepted: 28 December 2021 Published: 30 December 2021

Publisher's Note: MDPI stays neutral with regard to jurisdictional claims in published maps and institutional affiliations.

Copyright: (C) 2021 by the authors Licensee MDPI, Basel, Switzerland. This article is an open access article distributed under the terms and conditions of the Creative Commons Attribution (CC BY) license (https:// creativecommons.org/licenses/by/ $4.0 /)$

\begin{abstract}
Migraine headaches can be provoked by surgical stress and vasoactive effects of anesthetics of general anesthesia in the perioperative period. However, it is unclear whether general anesthesia increases the migraine risk after major surgery. Incidence and risk factors of postoperative migraine are also largely unknown. We utilized reimbursement claims data of Taiwan's National Health Insurance and performed propensity score matching analyses to compare the risk of postoperative migraine in patients without migraine initially who underwent general or neuraxial anesthesia. Multivariable logistic regressions were applied to calculate the adjusted odds ratio (aOR) and 95\% confidence interval (CI) for migraine risk. A total of 68,131 matched pairs were analyzed. The overall incidence of migraine was 9.82 per 1000 person-years. General anesthesia was not associated with a greater risk of migraine compared with neuraxial anesthesia (aORs: 0.93, 95\% CI: 0.80-1.09). This finding was consistent across subgroups of different migraine subtypes, uses of migraine medications, and varying postoperative periods. Influential factors for postoperative migraine were age (aOR: 0.99), sex (male vs. female, aOR: 0.50), pre-existing anxiety disorder (aOR: 2.43 ) or depressive disorder (aOR: 2.29), concurrent uses of systemic corticosteroids (aOR: 1.45), ephedrine (aOR: 1.45), and theophylline (aOR: 1.40), and number of emergency room visits before surgery. There was no difference in the risk of postoperative migraine between surgical patients undergoing general and neuraxial anesthesia. This study identified the risk factors for postoperative migraine headaches, which may provide an implication in facilitating early diagnoses and treatment.
\end{abstract}

Keywords: headache; postoperative; risk factor; spinal anesthesia; stress

\section{Introduction}

Migraine is a complex neurovascular disorder that affects an estimated $15 \%$ of people worldwide [1,2]. Despite the recent advances in diagnosis and treatment, migraine remains the second leading cause of disability globally and accounts for the financial costs of USD 23 billion and more than EUR 50 billion in the USA and Europe, respectively [1,2].

Surgical patients are predisposed to migraine headaches due to stress, mental tension, and bright lights in the perioperative period [3,4]. Postoperative migraine headaches can cause emotional distress, induce sleep disorders, and impair health-related quality of life 
in surgical patients [1,2]. In addition, studies showed that patients with migraine have increased risks of some perioperative complications, including postoperative nausea and vomiting, ischemic stroke, and rehospitalization [5,6]. A prospective cohort study showed that the diagnosis of migraine was significantly associated with increased long-term risks of major cardiovascular diseases and cardiovascular mortality in women [7]. More efforts are needed to implement and improve migraine diagnosis and care in the postoperative period.

One of the theories of migraine pathogenesis is stimuli from vasodilation in intracranial arteries (mainly the branches of the middle meningeal artery), which are innervated by trigeminal nerve, to nociceptors in arterioles [8,9]. In animal studies, volatile anesthetics were found to have a vasodilatory effect on meningeal arterioles [10,11], and this effect may serve as a trigger of migraine after general anesthesia. By contrast, some studies reported the use of Propofol as the treatment of refractory migraine [12,13]. The overall effect of general anesthesia on the precipitation of migraine is poorly understood so far.

Cases studies have proposed that general anesthesia and opioids may be related to the development of postoperative migraine headaches [14-18]. Although several risk factors were reported for postoperative headache, it remains unclear whether general anesthesia increases the risk of postoperative migraine compared with other forms of anesthesia [3,19-21]. In addition, risk factors for postoperative migraine episodes are also largely unknown in current literature. The diagnosis of perioperative migraine can be difficult and complicated to establish if clinicians are unable to identify high-risk patients $[14,16,17]$.

Accordingly, we utilized Taiwan's National Health Insurance (NHI) research database to conduct the nationwide population-based cohort study. There are two objectives in this study. First, we aimed to compare the risk of postoperative migraine headaches between patients undergoing general and neuraxial anesthesia. Second, we sought to determine the risk factors for postoperative migraine headaches. Based on the current evidence [10,11,14-18], we hypothesized that general anesthesia was associated with a higher risk of postoperative migraine headaches compared with neuraxial anesthesia.

\section{Materials and Methods}

\subsection{Source of Data}

The present study was approved by the Institutional Review Board of Taipei Medical University in Taiwan (TMU-JIRB-N202101005). Written informed consent was waived by the Institutional Review Board. All methods of this study were performed in accordance with the STROBE guidelines and regulations. Taiwan's National Health Insurance program was launched in March 1995 and provided insurance to more than 99\% of Taiwan's 23.4 million residents at the end of 2013. The NHI research database contains comprehensive data of the insured individuals, including demographic attributes (date of birth, sex, and residential location) and claims data (outpatient and inpatient care, medical diagnoses, prescriptions, and procedures). To protect personal privacy, a unique identification number is assigned to each beneficiary and enciphered before the data are released for research purposes. The NHI research database has been widely used in numerous epidemiological studies [22-25]. The present study used three Longitudinal Health Insurance Databases (LHID2000, LHID2005, and LHID2010), which randomly sampled 1 million beneficiaries from the original NHI research database in the years 2000, 2005, and 2010, respectively. The LHIDs contain the most updated medical claims of sampled beneficiaries since 1997. The representativeness of LHIDs has been validated by Taiwan's National Health Research Institutes [26].

\subsection{Study Population and Exposure Factors}

We used the medical claims of 3 million insured beneficiaries to select patients who underwent their first surgical procedures requiring general or neuraxial anesthesia with a length of hospital stay $\geq 2$ days in Taiwan between 1 January 2002 and 30 June 2013. We 
excluded surgeries which could only be performed with general anesthesia, patients who had any diagnoses of migraine in the outpatient or inpatient care within 24 months before the index surgery, and those who had a diagnosis of headache related to dural puncture or died within 180 days after the index surgery. Each subject with general anesthesia was randomly matched to a subject with neuraxial anesthesia in a ratio 1:1, using a frequency matched pair procedure.

\subsection{Outcome of Interest}

We identified patients who developed postoperative migraine headaches within 180 days after the index surgery by using the International Classification of Diseases, 9th Revision, Clinical Modification (ICD-9-CM) codes. (Supplementary Table S1) For sensitivity tests, migraine episodes in the varying postoperative periods $(30,60,90,120$, and 150 days) were also compared between groups. The diagnosis of migraine was made by board-certified neurologists. Uses of acute migraine medications within 180 days after surgery were also examined, including sumatriptan, rizatriptan, ergotamine, and dihydroergotamine [27].

\subsection{Covariates}

Surgical procedures were classified into orthopedic (lower limbs), genitourinary, anal, obstetric, and hernia repair surgeries. We used the ICD-9-CM codes of physicians' diagnoses within 24 months prior to surgery to ascertain the history of the following coexisting diseases, chosen based on data availability, physiological plausibility, and the existing literature: hypertension, diabetes mellitus, ischemic heart disease, atherosclerosis, heart failure, cerebrovascular disease, chronic kidney disease, chronic obstruction pulmonary disease, malignancy, anxiety disorder, depressive disorder, schizophrenia, and bipolar disorder [28]. Lifestyle factors included obesity, smoking disorder, alcohol use disorder, and malnutrition [28]. (Supplementary Table S1) Since adverse events after surgery may produce physical and emotional stress and trigger migraine headaches $[3,4]$, we also analyzed the major complications that occurred within 30 days after the index surgery, including pneumonia, septicemia, acute renal failure, pulmonary embolism, deep vein thrombosis, stroke, urinary tract infection, surgical site infection, acute myocardial infarction, cardiac dysrhythmias, and postoperative bleeding. Perioperative uses of blood transfusion [29-31] and need for intensive care [32] during the index surgical admission were analyzed. Our analyses also adjusted for the commonly used sympathomimetic drugs prescribed within 180 days after the index surgery, which might affect cerebral blood flow and modify migraine risk, including systemic corticosteroids, ephedrine, and theophylline [33-35].

\subsection{Statistical Analysis}

A non-parsimonious multivariable logistic regression model was applied to estimate a propensity score for subjects undergoing general or neuraxial anesthesia. Each subject with general anesthesia was matched to a subject with neuraxial anesthesia using a greedy matching algorithm within a tolerance limit of 0.05 and without replacement to adjust for age, sex, insurance premium, types of surgery, comorbidities, lifestyle factors, concurrent sympathomimetic drugs, number of hospitalizations, and number of emergency room visits within 24 months before the index surgery. Categorical variables were expressed using frequency and percentage, and continuous variables were summarized using mean and standard deviation. The distributions of baseline attributes in propensity-score matched samples were compared between groups by using standardized difference [36]. Multivariable logistic regressions models were used to calculate the adjusted odds ratio (aOR) and $95 \%$ confidence interval (CI) of postoperative migraine headaches. We considered a two-sided level of 0.05 statistically significant. All the statistical analyses were conducted using Statistics Analysis System (SAS), Version 9.4 (SAS Institute Inc., Cary, NC, USA). 


\section{Results}

\subsection{Baseline Patient Characteristics}

After meeting the patient selection criteria, the matching procedure generated 68,131 matched pairs with 66,989 person-years of follow-up for analyses. (Figure 1) Table 1 shows the baseline characteristics of the included subjects undergoing general or neuraxial anesthesia. The distributions of demographics, types of surgery, comorbidities, lifestyle factors, concurrent sympathomimetic drugs, number of hospitalizations, and number of emergency room visits were well balanced after matching.

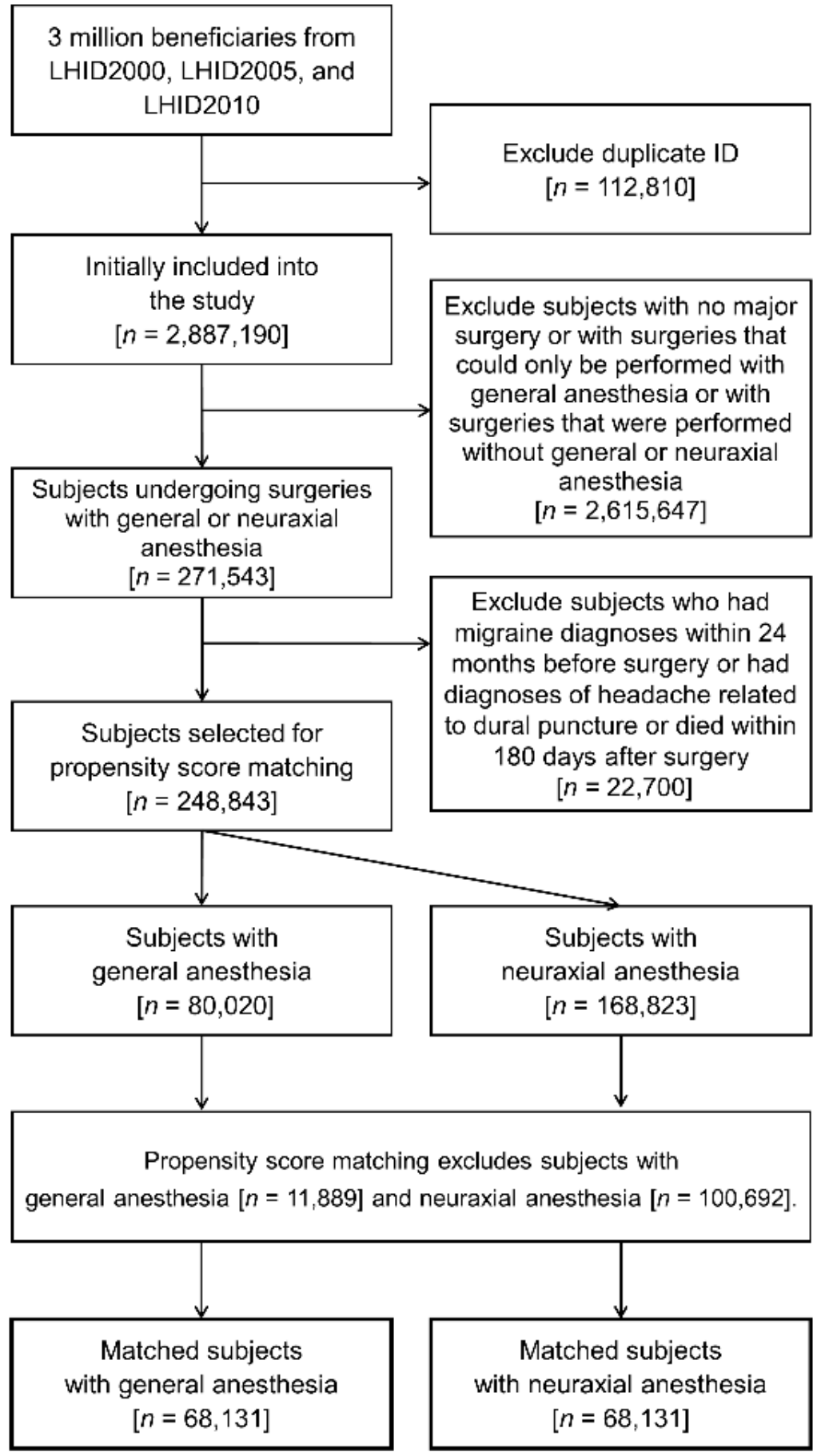

Figure 1. Flow diagram for patient selection. 
Table 1. Baseline characteristics of patients undergoing general and neuraxial anesthesia.

\begin{tabular}{|c|c|c|c|c|c|}
\hline \multirow{2}{*}{$\begin{array}{l}\text { Baseline Characteristic } \\
\text { Age (years), mean (SD) }\end{array}$} & \multicolumn{2}{|c|}{$\begin{array}{c}\text { General Anesthesia } \\
\qquad n=68,131\end{array}$} & \multicolumn{2}{|c|}{$\begin{array}{l}\text { Neuraxial Anesthesia } \\
\qquad n=68,131\end{array}$} & \multirow{2}{*}{$\begin{array}{c}\text { SDD } \\
0.0153\end{array}$} \\
\hline & 51.4 & 19.8 & 51.1 & 19.5 & \\
\hline Sex, male, $n(\%)$ & 36,890 & 54.2 & 37,003 & 54.3 & -0.0037 \\
\hline $\begin{array}{l}\text { Insurance premium (USD/month), } \\
n(\%)\end{array}$ & & & & & 0.0028 \\
\hline $0-500$ & 29,888 & 43.9 & 30,182 & 44.3 & \\
\hline $501-800$ & 22,810 & 33.5 & 22,375 & 32.8 & \\
\hline$\geq 801$ & 15,433 & 22.7 & 15,574 & 22.9 & \\
\hline \multicolumn{6}{|l|}{ Type of surgery, $n(\%)$} \\
\hline Orthopedic, lower limbs & 29,824 & 43.8 & 29,629 & 43.5 & 0.0064 \\
\hline Genitourinary & 18,018 & 26.5 & 17,692 & 26.0 & 0.0136 \\
\hline Anal & 7373 & 10.8 & 7613 & 11.2 & -0.0198 \\
\hline Obstetric & 6972 & 10.2 & 6966 & 10.2 & 0.0005 \\
\hline Hernia repair & 6168 & 9.1 & 6506 & 9.6 & -0.0324 \\
\hline \multicolumn{6}{|l|}{ Lifestyle factors, $n(\%)$} \\
\hline Obesity & 371 & 0.5 & 381 & 0.6 & -0.0147 \\
\hline Smoking disorder & 434 & 0.6 & 437 & 0.6 & -0.0038 \\
\hline Alcohol use disorder & 945 & 1.4 & 925 & 1.4 & 0.0120 \\
\hline Malnutrition & 477 & 0.7 & 486 & 0.7 & -0.0104 \\
\hline \multicolumn{6}{|l|}{ Comorbidity, $n(\%)$} \\
\hline Hypertension & 20,416 & 30.0 & 20,149 & 29.6 & 0.0103 \\
\hline Diabetes mellitus & 9719 & 14.3 & 9542 & 14.0 & 0.0118 \\
\hline Ischemic heart disease & 7363 & 10.8 & 7357 & 10.8 & 0.0005 \\
\hline Atherosclerosis & 604 & 0.9 & 594 & 0.9 & 0.0093 \\
\hline Heart failure & 2242 & 3.3 & 2212 & 3.3 & 0.0077 \\
\hline Cerebrovascular disease & 5453 & 8.0 & 5429 & 8.0 & 0.0026 \\
\hline Chronic kidney disease & 3708 & 5.4 & 3692 & 5.4 & 0.0025 \\
\hline COPD & 5663 & 8.3 & 5685 & 8.3 & -0.0023 \\
\hline Malignancy & 4478 & 6.6 & 4446 & 6.5 & 0.0042 \\
\hline Anxiety disorder & 7127 & 10.5 & 7131 & 10.5 & -0.0003 \\
\hline Depressive disorder & 723 & 1.1 & 734 & 1.1 & -0.0084 \\
\hline Schizophrenia & 383 & 0.6 & 374 & 0.6 & 0.0132 \\
\hline Bipolar disorder & 268 & 0.4 & 281 & 0.4 & -0.0262 \\
\hline \multicolumn{6}{|l|}{$\begin{array}{l}\text { Concurrent sympathomimetic drugs, } \\
n(\%)\end{array}$} \\
\hline Systemic corticosteroids & 11,439 & 16.8 & 11,291 & 16.6 & 0.0086 \\
\hline Ephedrine & 11,961 & 17.6 & 12,099 & 17.8 & -0.0077 \\
\hline Theophylline & 6623 & 9.7 & 6702 & 9.8 & -0.0072 \\
\hline Number of hospitalizations, $n(\%)$ & & & & & 0.0238 \\
\hline 0 & 52,007 & 76.3 & 52,997 & 77.8 & \\
\hline 1 & 10,502 & 15.4 & 9750 & 14.3 & \\
\hline 2 & 3217 & 4.7 & 2934 & 4.3 & \\
\hline$\geq 3$ & 2405 & 3.5 & 2450 & 3.6 & \\
\hline Number of ER visits, $n(\%)$ & & & & & -0.0004 \\
\hline 0 & 39,554 & 58.1 & 39,772 & 58.4 & \\
\hline 1 & 16,202 & 23.8 & 15,864 & 23.3 & \\
\hline 2 & 6423 & 9.4 & 6421 & 9.4 & \\
\hline$\geq 3$ & 5952 & 8.7 & 6074 & 8.9 & \\
\hline Postoperative complications, $n(\%)$ & 8533 & 12.5 & 9105 & 13.4 & -0.0411 \\
\hline Blood transfusion, $n(\%)$ & 986 & 1.5 & 815 & 1.2 & 0.1064 \\
\hline ICU admission, $n(\%)$ & 430 & 0.6 & 374 & 0.6 & 0.0774 \\
\hline
\end{tabular}

Abbreviation: COPD = chronic obstruction pulmonary disease; ER = emergency room; ICU = intensive care unit $\mathrm{SD}=$ standard deviation; SDD = standardized difference; and USD = United States dollar.

\subsection{Risk of Postoperative Migraine Headaches}

In the matched cohort, 658 patients developed a new-onset migraine during the halfyear follow-up, and 318 and 340 after general and neuraxial anesthesia, respectively. The overall incidence of migraine was 9.82 per 1000 person-years, and 9.49 and 10.15 for patients undergoing general and neuraxial anesthesia, respectively.

Table 2 shows the results of univariate and multivariable logistic regression models for the risk of postoperative migraine headaches. There was no significant difference in the migraine risk between general and neuraxial anesthesia, aOR: 0.93 (95\% CI: 0.80-1.09). Independent influential factors for migraine were age (aOR: 0.99), sex (male vs. female, aOR: 0.50), pre-existing anxiety disorder (aOR: 2.43), depressive disorder (aOR: 2.29), con- 
current uses of systemic corticosteroids (aOR: 1.45), ephedrine (aOR: 1.45), and theophylline (aOR: 1.40), and number of emergency room visits before surgery ( 1 vs. 0 , aOR: $1.12 ; 2$ vs. 0 , aOR: $1.14 ; \geq 3$ vs. 0, aOR: 1.68$)$.

Table 2. Univariate and multivariable analyses for the risk of postoperative migraine.

\begin{tabular}{|c|c|c|c|c|c|c|}
\hline & \multicolumn{3}{|c|}{ Univariate } & \multicolumn{3}{|c|}{ Multivariable } \\
\hline & cOR & $95 \%$ CI & $p$ & aOR & $95 \%$ CI & $p$ \\
\hline General vs. neuraxial anesthesia & 0.94 & $0.80-1.09$ & 0.3900 & 0.93 & $0.80-1.09$ & 0.3578 \\
\hline Age (years) & 1.00 & $1.00-1.00$ & 0.8573 & 0.99 & $0.99-1.00$ & 0.0211 \\
\hline Sex, male & 0.51 & $0.44-0.60$ & $<0.0001$ & 0.50 & $0.42-0.60$ & $<0.0001$ \\
\hline Insurance premium (USD/month) & & & 0.5394 & & & 0.7854 \\
\hline $501-800$ vs. $0-500$ & 0.98 & $0.83-1.17$ & 0.6495 & 0.97 & $0.81-1.16$ & 0.9443 \\
\hline$\geq 801$ vs. $0-500$ & 0.89 & $0.73-1.09$ & 0.2815 & 0.93 & $0.75-1.15$ & 0.5540 \\
\hline \multicolumn{7}{|l|}{ Type of surgery } \\
\hline Orthopedic, lower limbs & 0.88 & $0.75-1.03$ & 0.1136 & 0.43 & $0.06-3.14$ & 0.4079 \\
\hline Genitourinary & 0.95 & $0.80-1.13$ & 0.5671 & 0.52 & $0.07-3.70$ & 0.5096 \\
\hline Anal & 1.32 & $1.06-1.64$ & 0.0143 & 0.62 & $0.09-4.49$ & 0.6356 \\
\hline Obstetric & 1.20 & $0.95-1.52$ & 0.1318 & 0.39 & $0.05-2.88$ & 0.3592 \\
\hline Hernia repair & 0.89 & $0.67-1.17$ & 0.4044 & 0.59 & $0.08-4.23$ & 0.6000 \\
\hline \multicolumn{7}{|l|}{ Lifestyle factors } \\
\hline Obesity & 1.66 & $0.74-3.73$ & 0.2164 & 1.30 & $0.58-2.93$ & 0.5261 \\
\hline Smoking disorder & 1.44 & $0.64-3.22$ & 0.3778 & 1.50 & $0.66-3.37$ & 0.3327 \\
\hline Alcohol use disorder & 0.55 & $0.23-1.33$ & 0.1823 & 0.51 & $0.21-1.24$ & 0.1359 \\
\hline Malnutrition & 1.08 & $0.45-2.60$ & 0.8655 & 0.84 & $0.35-2.05$ & 0.7078 \\
\hline \multicolumn{7}{|l|}{ Comorbidity } \\
\hline Hypertension & 1.11 & $0.95-1.31$ & 0.1966 & 0.92 & $0.75-1.14$ & 0.4612 \\
\hline Diabetes mellitus & 1.12 & $0.90-1.38$ & 0.3135 & 1.03 & $0.82-1.30$ & 0.8100 \\
\hline Ischemic heart disease & 1.38 & $1.11-1.72$ & 0.0041 & 1.12 & $0.87-1.45$ & 0.3697 \\
\hline Atherosclerosis & 1.57 & $0.81-3.03$ & 0.1820 & 1.26 & $0.65-2.47$ & 0.4948 \\
\hline Heart failure & 1.32 & $0.90-1.93$ & 0.1538 & 1.02 & $0.67-1.53$ & 0.9442 \\
\hline Cerebrovascular disease & 1.31 & $1.02-1.69$ & 0.0374 & 1.20 & $0.90-1.60$ & 0.2110 \\
\hline Chronic kidney disease & 1.37 & $1.02-1.84$ & 0.0352 & 1.04 & $0.76-1.43$ & 0.8015 \\
\hline COPD & 1.38 & $1.08-1.76$ & 0.0104 & 1.16 & $0.89-1.51$ & 0.2772 \\
\hline Malignancy & 0.92 & $0.67-1.27$ & 0.6252 & 0.93 & $0.67-1.30$ & 0.6651 \\
\hline Anxiety disorder & 3.05 & $2.56-3.64$ & $<0.0001$ & 2.43 & $2.01-2.95$ & $<0.0001$ \\
\hline Depressive disorder & 4.18 & $2.85-6.12$ & $<0.0001$ & 2.29 & $1.53-3.44$ & $<0.0001$ \\
\hline Schizophrenia & 1.10 & $0.41-2.94$ & 0.8563 & 0.86 & $0.31-2.33$ & 0.7588 \\
\hline Bipolar disorder & 1.13 & $0.36-3.53$ & 0.8296 & 0.51 & $0.16-1.63$ & 0.2555 \\
\hline \multicolumn{7}{|l|}{ Concurrent sympathomimetic drugs } \\
\hline Systemic corticosteroids & 1.69 & $1.42-2.02$ & $<0.0001$ & 1.45 & $1.21-1.74$ & $<0.0001$ \\
\hline Ephedrine & 1.80 & $1.52-2.14$ & $<0.0001$ & 1.45 & $1.21-1.75$ & $<0.0001$ \\
\hline Theophylline & 1.84 & $1.50-2.26$ & $<0.0001$ & 1.40 & $1.12-1.74$ & 0.0036 \\
\hline Number of hospitalizations & & & 0.1994 & & & 0.2011 \\
\hline 1 vs. 0 & 1.24 & $1.01-1.52$ & 0.2302 & 1.05 & $0.85-1.30$ & 0.0668 \\
\hline 2 vs. 0 & 1.16 & $0.82-1.65$ & 0.7173 & 0.87 & $0.60-1.27$ & 0.9935 \\
\hline$\geq 3$ vs. 0 & 1.02 & $0.67-1.56$ & 0.6570 & 0.64 & $0.40-1.01$ & 0.0676 \\
\hline Number of ER visits & & & $<0.0001$ & & & 0.0012 \\
\hline 1 vs. 0 & 1.13 & $0.93-1.37$ & 0.1220 & 1.12 & $0.92-1.36$ & 0.3045 \\
\hline 2 vs. 0 & 1.20 & $0.92-1.56$ & 0.5957 & 1.14 & $0.87-1.50$ & 0.5517 \\
\hline$\geq 3$ vs. 0 & 1.89 & $1.51-2.37$ & $<0.0001$ & 1.68 & $1.30-2.16$ & 0.0004 \\
\hline Postoperative complications & 1.03 & $0.82-1.29$ & 0.8300 & 0.92 & $0.73-1.17$ & 0.5028 \\
\hline Blood transfusion & 0.69 & $0.31-1.54$ & 0.3608 & 0.58 & $0.24-1.39$ & 0.2217 \\
\hline ICU admission & 1.29 & $0.54-3.12$ & 0.5686 & 1.55 & $0.59-4.06$ & 0.3725 \\
\hline
\end{tabular}

Abbreviation: aOR = adjusted odds ratio; COPD = chronic obstruction pulmonary disease; $\mathrm{cOR}=$ crude odds ratio; ER = emergency room; ICU = intensive care unit; USD = United States dollar.

\subsection{Subgroup Analyses}

There was no significant difference in the migraine risk between patients undergoing general or neuraxial anesthesia across the subgroups of different migraine subtypes, uses 
of migraine medications, or varying postoperative periods. (Table 3) There was no difference between general and neuraxial anesthesia in migraine risk across the subgroups of age $\geq$ or $<65$ years, sex, anxiety disorder, depressive disorder, concurrent uses of systemic corticosteroids, ephedrine, theophylline, postoperative complications, or admission to intensive care unit, either (Table 4).

Table 3. Risk of postoperative migraine stratified by migraine subtypes, migraine medications, and varying postoperative periods.

\begin{tabular}{|c|c|c|c|c|c|c|c|c|c|}
\hline & \multicolumn{3}{|c|}{ General Anesthesia } & \multicolumn{3}{|c|}{ Neuraxial Anesthesia } & \multicolumn{3}{|c|}{ Migraine Risk } \\
\hline & Event & Rate (\%) & $\begin{array}{c}\text { Crude } \\
\text { Incidence } \\
\text { Rate/1000 PY }\end{array}$ & Event & Rate (\%) & $\begin{array}{c}\text { Crude } \\
\text { Incidence } \\
\text { Rate/1000 PY }\end{array}$ & $\begin{array}{l}\text { Incidence } \\
\text { Rate Ratio }\end{array}$ & aOR $(95 \% \mathrm{CI}) \dagger$ & $p$ \\
\hline All migraine & 318 & 0.47 & 9.49 & 340 & 0.50 & 10.15 & 0.94 & $0.93(0.80-1.09)$ & 0.3578 \\
\hline Migraine with aura & 32 & 0.05 & 0.96 & 31 & 0.05 & 0.93 & 1.03 & $1.02(0.62-1.68)$ & 0.9295 \\
\hline Migraine without aura & 58 & 0.09 & 1.73 & 78 & 0.11 & 2.33 & 0.74 & $0.73(0.52-1.03)$ & 0.0697 \\
\hline Migraine, unspecified & 228 & 0.33 & 6.81 & 231 & 0.34 & 6.90 & 0.99 & $0.99(0.82-1.19)$ & 0.8857 \\
\hline $\begin{array}{l}\text { Migraine with } \\
\text { medications }\end{array}$ & 102 & 0.15 & 3.05 & 83 & 0.12 & 2.48 & 1.23 & $1.23(0.92-1.64)$ & 0.1717 \\
\hline 30-day migraine & 60 & 0.09 & 10.73 & 58 & 0.09 & 10.37 & 1.03 & $1.03(0.72-1.48)$ & 0.8706 \\
\hline 60-day migraine & 115 & 0.17 & 10.28 & 112 & 0.16 & 10.02 & 1.03 & $1.02(0.79-1.33)$ & 0.8793 \\
\hline 90-day migraine & 174 & 0.26 & 10.38 & 161 & 0.24 & 9.60 & 1.08 & $1.08(0.87-1.34)$ & 0.4981 \\
\hline 120-day migraine & 218 & 0.32 & 9.76 & 220 & 0.32 & 9.84 & 0.99 & $0.99(0.82-1.19)$ & 0.8893 \\
\hline 150-day migraine & 276 & 0.41 & 9.88 & 282 & 0.41 & 10.10 & 0.98 & $0.97(0.82-1.15)$ & 0.7570 \\
\hline
\end{tabular}

Abbreviation: aOR $=$ adjusted odds ratio; $\mathrm{CI}=$ confidence interval; $\mathrm{PY}=$ person-years. + Adjusted for age (continuous), sex, insurance premium (categorical), types of surgery, comorbidities, lifestyle factors, concurrent sympathomimetic drugs, number of hospitalizations, number of emergency room visits, postoperative complications, perioperative uses of blood transfusion, and intensive care unit care.

Table 4. Subgroup analyses of postoperative migraine for patients undergoing general and neuraxial anesthesia.

\begin{tabular}{|c|c|c|c|c|c|c|}
\hline Subgroup & & $n$ & Event & Rate (\%) & $\operatorname{aOR}(95 \% \mathrm{CI})+$ & $p$ \\
\hline \multirow[t]{2}{*}{ Age $\geq 65$ years } & GA & 19,894 & 77 & 0.39 & $0.94(0.69-1.29)$ & 0.6985 \\
\hline & NA & 20,074 & 83 & 0.41 & reference & \\
\hline \multirow[t]{2}{*}{ Age $<65$ years } & GA & 48,237 & 241 & 0.50 & $0.93(0.78-1.11)$ & 0.3972 \\
\hline & NA & 48,057 & 257 & 0.53 & reference & \\
\hline \multirow[t]{2}{*}{ Male } & GA & 36,890 & 112 & 0.30 & $0.84(0.65-1.08)$ & 0.1672 \\
\hline & NA & 37,003 & 136 & 0.37 & reference & \\
\hline \multirow[t]{2}{*}{ Female } & GA & 31,241 & 206 & 0.66 & $0.98(0.81-1.20)$ & 0.8629 \\
\hline & NA & 31,128 & 204 & 0.66 & reference & \\
\hline \multirow[t]{2}{*}{ Anxiety disorder } & GA & 7127 & 77 & 1.08 & $0.80(0.59-1.08)$ & 0.1410 \\
\hline & NA & 7131 & 95 & 1.33 & reference & \\
\hline \multirow[t]{2}{*}{ No anxiety disorder } & GA & 61,004 & 241 & 0.40 & $0.98(0.82-1.18)$ & 0.8551 \\
\hline & NA & 61,000 & 245 & 0.40 & reference & \\
\hline \multirow[t]{2}{*}{ Depressive disorder } & GA & 723 & 13 & 1.80 & $0.79(0.34-1.83)$ & 0.5818 \\
\hline & NA & 734 & 15 & 2.04 & reference & \\
\hline \multirow[t]{2}{*}{ No depressive disorder } & GA & 67,408 & 305 & 0.45 & $0.94(0.80-1.10)$ & 0.4096 \\
\hline & NA & 67,397 & 325 & 0.48 & reference & \\
\hline \multirow{2}{*}{$\begin{array}{l}\text { Use of systemic } \\
\text { corticosteroids }\end{array}$} & GA & 11,439 & 82 & 0.72 & $0.98(0.72-1.33)$ & 0.8769 \\
\hline & NA & 11,291 & 84 & 0.74 & reference & \\
\hline \multirow{2}{*}{$\begin{array}{l}\text { No use of systemic } \\
\text { corticosteroids }\end{array}$} & GA & 56,692 & 236 & 0.42 & $0.92(0.77-1.09)$ & 0.3280 \\
\hline & NA & 56,840 & 256 & 0.45 & reference & \\
\hline \multirow[t]{2}{*}{ Use of ephedrine } & GA & 11,961 & 84 & 0.70 & $0.86(0.64-1.15)$ & 0.3119 \\
\hline & NA & 12,099 & 99 & 0.82 & reference & \\
\hline \multirow[t]{2}{*}{ No use of ephedrine } & GA & 56,170 & 234 & 0.42 & $0.96(0.80-1.15)$ & 0.6469 \\
\hline & NA & 56,032 & 241 & 0.43 & reference & \\
\hline \multirow[t]{2}{*}{ Use of theophylline } & GA & 6623 & 48 & 0.72 & $0.78(0.53-1.14)$ & 0.1996 \\
\hline & NA & 6702 & 61 & 0.91 & reference & \\
\hline
\end{tabular}


Table 4. Cont.

\begin{tabular}{ccccccc}
\hline Subgroup & & $n$ & Event & Rate (\%) & aOR (95\% CI) $\dagger$ & $p$ \\
\hline No use of theophylline & GA & 61,508 & 270 & 0.44 & $0.96(0.81-1.14)$ & 0.6500 \\
& NA & 61,429 & 279 & 0.45 & reference & \\
Postoperative & & & & & & \\
complications & GA & 8533 & 41 & 0.48 & $0.94(0.61-1.43)$ & 0.7581 \\
complications & & & & & & \\
& NA & 9105 & 46 & 0.51 & reference & \\
No postoperative & GA & 59,598 & 277 & 0.46 & $0.93(0.79-1.10)$ & 0.3777 \\
complications & NA & 59,026 & 294 & 0.50 & reference & \\
Admission to ICU & GA & 430 & 3 & 0.70 & $0.97(0.08-12.33)$ & 0.9825 \\
& NA & 374 & 2 & 0.53 & reference & \\
No admission to ICU & GA & 67,701 & 315 & 0.47 & $0.93(0.79-1.08)$ & 0.3357 \\
& NA & 67,757 & 338 & 0.50 & reference & \\
\hline
\end{tabular}

Abbreviation: aOR = adjusted odds ratio; $\mathrm{CI}=$ confidence interval; $\mathrm{GA}$ = general anesthesia; ICU = intensive care unit; NA = neuraxial anesthesia. + Adjusted for age (continuous), sex, insurance premium (categorical), types of surgery, comorbidities, lifestyle factors, concurrent sympathomimetic drugs, number of hospitalizations, number of emergency room visits, postoperative complications, perioperative uses of blood transfusion, and intensive care unit care.

\section{Discussion}

In this study, we found that there was no significant difference in the risk of postoperative migraine headaches between patients undergoing general or neuraxial anesthesia. The results were consistent across subgroups of different migraine subtypes, uses of migraine medications, and varying postoperative periods. Importantly, our analyses identified several risk factors for postoperative migraine headaches, including younger age, female, pre-existing anxiety disorder, depressive disorder, and a greater number of preoperative emergency visits. The perioperative uses of systemic corticosteroid, ephedrine, and theophylline were associated with increased risks of migraine after surgery. To our knowledge, this is the first large-scale study to evaluate the overall incidence and potential risk factors for migraine headaches after major surgery. These findings provide evidence for the diagnosis, risk-stratification, and treatment of migraine headaches after major surgery.

Epidemiological study has reported that overall incidence of migraine was estimated at 8.1 per 1000 person-years in people without migraine initially [37]. In our study, the incidence of postoperative migraine headaches was 9.82 per 1000 person-years in the half-year follow-up, which was $21 \%$ higher than that of previous reports in general population [37]. Patients in our cohort were under the stress of surgery, which has been identified as the most important trigger of migraine [3,4,38]. Moreover, the present study showed that patients with pre-existing anxiety or depressive disorder had a greater risk of developing postoperative migraine. Emotional disturbances were found commonly comorbid with migraine in the general population [39,40]. The causation between anxiety/depressive disorders and migraine was unclear in the perioperative period. It is also uncertain whether the stress of surgery modifies the migraine risk among patients with mental health disorders. Our analyses revealed that more emergency room visits prior to surgery were associated with a higher risk of postoperative migraine. Two observational studies demonstrated a relationship between emergency room visits and severity of migraine [41,42]. In our study, preoperative frequency of emergency visits might reflect disease severity and surgical urgency, which potentially underlay the higher risk of postoperative migraine.

Our results refuted the primary hypothesis that general anesthesia was associated with a higher risk of postoperative migraine compared with neuraxial anesthesia. Until now, there are only few case studies reporting migraine headaches after general anesthesia and fentanyl sedation [14-18]. The association between general anesthesia and postoperative migraine episodes has been hypothesized, but there is still no study to compare the migraine risk between general anesthesia and other types of anesthesia. We raised the following possible explanations for our results. First, although the vasodilatory effect of volatile 
anesthetics on meningeal arterioles has been proposed to trigger migraine headaches $[10,11]$, this neurovascular effect may be outweighed by Propofol used in general anesthesia. Propofol is an agonist of gamma-aminobutyric acid receptors, which inhibit the activity of central serotonergic neurons in the raphe nuclei and theoretically exert anti-migraine effects [12,13]. In addition, Propofol reduces both cerebral blood flow and cerebral metabolic rate, which may prevent the development of migraine [12,13]. Future studies are needed to investigate the potential impact of different regimens of general anesthesia on the risk of postoperative migraine, such as Propofol-based total intravenous anesthesia and opioidfree general anesthesia.

Our results suggested that uses of systemic corticosteroid, ephedrine, and theophylline were correlated with increased migraine risks. First, systemic corticosteroid has been used as an acute migraine treatment, and it is particularly effective for patients with refractory migraine, a history of recurrent headaches, and status migrainosus [43-45]. In our study, the use of systemic corticosteroid may represent a rescue therapy for migraine in these patients. Second, ephedrine is a non-selective beta agonist with alpha-1 activity [46,47]. In the current practice guideline, beta blockers without intrinsic sympathomimetic activity are used to prevent recurrent migraine headaches [46]. In a previous study conducted on healthy males, intravenous infusion of ephedrine caused an increase in blood flow of external carotid artery [47]. We reasoned that the increased blood flow of external carotid artery may increase the blood flow and induce vasodilation in the branches of middle meningeal artery, causing stimuli to trigeminal nerve and thereby triggering migraine [8,9]. Third, theophylline is a phosphodiesterase inhibiting agent used as the therapy for chronic obstructive pulmonary disease and asthma [48]. Theophylline has a narrow therapeutic window and its overdose can stimulate the central nervous system and cause headaches, insomnia, and seizure [49]. Besides, previous studies have shown that patients with asthma had a higher prevalence and incidence of migraine [50,51]. However, the mechanism is still unknown. Future studies are warranted to investigate the potential neurophysiological effects of theophylline on migraine headaches.

The present study discovered some potentially modifiable factors for migraine headaches after surgery, including systemic corticosteroids, ephedrine, and theophylline. Whether avoiding these drugs prevents the occurrence of migraine remains to be seen. It is also unclear what proportion of postoperative migraine progresses to chronic migraine. To date, the proven effective treatment for chronic migraine includes oral medications, neuromodulation, and nerve blockade [28,52]. Meta-analyses showed that acupuncture as a treatment modality was associated with a significant reduction in both headache frequency and response compared with routine care only and with prophylactic drugs at 2 months [53]. However, these results were potentially limited by the heterogeneity across studies and placebo effects [53]. Future studies are warranted to better evaluate the potential benefits and adverse effects of acupuncture in the prevention and treatment of chronic migraine.

There were some limitations to our study. First, our data did not contain information about physical measures (e.g., blood pressure), biochemical laboratory tests (e.g., stress hormone levels), socioeconomic factors (e.g., education level), and clinical data on detailed surgical (elective, urgent or emergent surgery, and duration of surgery) and anesthetic management (uses of vasoconstrictors or vasodilators, types and doses of anesthetics and opioids) that were not covered by NHI database. Second, our analyses have excluded patients who had a diagnosis of chronic migraine before surgery and therefore it is unclear whether types of anesthesia affect the precipitation of migraine headaches in this population. Third, surgeries that could only be performed with general anesthesia (e.g., brain surgery and ear, nose, and throat surgery) were excluded from analyses, which might decrease the generalizability of study results [32]. Fourth, a recent study reported that pre-existing migraine was a risk factor for postoperative nausea and vomiting [5]. However, primary care physicians rarely used diagnosis codes to document postoperative nausea and vomiting in the NHI research database probably due to its transient and mild 
symptoms compared with active surgical problems. This precluded meaningful analyses of the relationship between migraine headaches and postoperative nausea and vomiting in this study. Fifth, our dataset did not include patients with migraine who did not seek conventional medical care due to mild symptoms. Sixth, we did not evaluate the risk of migraine among people who did not undergo surgery or anesthesia. Finally, our cohort was only followed up until the end of 2013, due to the regulations of the NHI research database.

\section{Conclusions}

There was no significant difference in the incidence of postoperative migraine headaches in patients undergoing general or neuraxial anesthesia for surgeries that could be performed with both techniques. Several risk factors for migraine were determined, including younger age, female, pre-existing depressive and anxiety disorders, concurrent uses of systemic corticosteroids, ephedrine, and theophylline and number of emergency room visits before surgery. These findings may provide an implication for early diagnoses and prompt interventions of postoperative migraine headaches. More studies are needed to diagnose and evaluate postoperative migraines among patients receiving different anesthetics in the immediate postoperative period.

Supplementary Materials: The following are available online at https:/ /www.mdpi.com/article/10 .3390/ijerph19010362/s1, Table S1: ICD-9-CM codes of covariates and outcomes.

Author Contributions: Conceptualization, C.-Y.L., C.-C.L. and Y.-H.T.; methodology, Y.-H.T.; software, Y.-H.T.; validation, H.-Y.L., H.-L.W. and W.-C.L.; formal analysis, Y.-H.T.; investigation, W.-C.L. and Y.-H.T.; resources, J.-T.C. and Y.-G.C.; data curation, T.-J.C. and Y.-X.D.; writing-original draft preparation, C.-Y.L. and C.-C.L.; writing-review and editing, H.-Y.L., J.-T.C., Y.-G.C., T.-J.C., Y.-X.D., H.-L.W. and Y.-H.T.; visualization, Y.-H.T.; supervision, W.-C.L. and Y.-H.T.; project administration, Y.-H.T.; and funding acquisition, Y.-H.T., W.-C.L. and Y.-H.T. contributed equally to this work. All authors have read and agreed to the published version of the manuscript.

Funding: This research was funded by Taipei Medical University, Taiwan, grant number TMU110-AE1-B11. The APC was funded by Shuang Ho Hospital, Taipei Medical University, New Taipei City, Taiwan.

Institutional Review Board Statement: The study was conducted according to the guidelines of the Declaration of Helsinki, and approved by the Institutional Review Board of Taipei Medical University (TMU-JIRB-N202101005).

Informed Consent Statement: Patient consent was waived by the Institutional Review Board.

Data Availability Statement: The data presented in this study are available on request from the corresponding author.

Conflicts of Interest: The authors declare no conflict of interest.

\section{References}

1. Ashina, M.; Katsarava, Z.; Do, T.P.; Buse, D.C.; Pozo-Rosich, P.; Özge, A.; Krymchantowski, A.V.; Lebedeva, E.R.; Ravishankar, K.; Yu, S.; et al. Migraine: Epidemiology and systems of care. Lancet 2021, 397, 1485-1495. [CrossRef]

2. Burch, R.C.; Buse, D.C.; Lipton, R.B. Migraine: Epidemiology, Burden, and Comorbidity. Neurol. Clin. 2019, 37, 631-649. [CrossRef] [PubMed]

3. Faithfull, N.S. Post-operative headache-A multifactorial analysis. Eur. J. Anaesthesiol. 1991, 8, 59-63.

4. Ulrich, V.; Olesen, J.; Gervil, M.; Russell, M.B. Possible Risk Factors and Precipitants for Migraine with Aura in Discordant Twin-Pairs: A Population-Based Study. Cephalalgia 2000, 20, 821-825. [CrossRef]

5. Kim, J.-H.; Lim, M.-S.; Lee, S.-H.; Kwon, Y.-S.; Lee, J.J.; Sohn, J.-H. Consideration of Migraines among Risk Factors for Postoperative Nausea and Vomiting. J. Clin. Med. 2020, 9, 3154. [CrossRef]

6. Timm, F.P.; Houle, T.; Grabitz, S.D.; Lihn, A.-L.; Stokholm, J.B.; Eikermann-Haerter, K.; Nozari, A.; Kurth, T.; Eikermann, M. Migraine and risk of perioperative ischemic stroke and hospital readmission: Hospital based registry study. BMJ 2017, $356, \mathrm{i} 6635$. [CrossRef]

7. Kurth, T.; Winter, A.C.; Eliassen, A.H.; Dushkes, R.; Mukamal, K.J.; Rimm, E.B.; Willett, W.C.; E Manson, J.; Rexrode, K. Migraine and risk of cardiovascular disease in women: Prospective cohort study. BMJ 2016, 353, i2610. [CrossRef] 
8. Levy, D.; Ramírez, A.L.; MaassenVanDenBrink, A. Current understanding of meningeal and cerebral vascular function underlying migraine headache. Cephalalgia 2018, 39, 1606-1622. [CrossRef] [PubMed]

9. Ashina, M. Migraine. N. Engl. J. Med. 2020, 383, 1866-1876. [CrossRef]

10. Iida, H.; Ohata, H.; Iida, M.; Watanabe, Y.; Dohi, S. Isoflurane and Sevoflurane Induce Vasodilation of Cerebral Vessels via ATP-sensitive $\mathrm{K}^{+}$Channel Activation. Anesthesiol. 1998, 89, 954-960. [CrossRef]

11. Inoue, S.; Kawaguchi, M.; Kurehara, K.; Sakamoto, T.; Kishi, K.; Einaga, T.; Kitaguchi, K.; Furuya, H. Mild hypothermia can enhance pial arteriolar vasodilation induced by isoflurane and sevoflurane in cats. Crit. Care Med. 2002, 30, 1863-1869. [CrossRef]

12. Dhir, A. Propofol in the treatment of refractory migraine headaches. Expert Rev. Neurother. 2016, 16, 1007-1011. [CrossRef] [PubMed]

13. Piatka, C.; Beckett, R.D. Propofol for Treatment of Acute Migraine in the Emergency Department: A Systematic Review. Acad. Emerg. Med. 2020, 27, 148-160. [CrossRef] [PubMed]

14. Thurlow, J.A. Hemiplegia following general anaesthesia: An unusual presentation of migraine. Eur. J. Anaesthesiol. 1998, 15, 610-612. [CrossRef]

15. Gil-Gouveia, R.; Wilkinson, P.A.; Kaube, H. Severe hemiplegic migraine attack precipitated by fentanyl sedation for esophagogastroscopy. Neurology 2004, 63, 2446-2447. [CrossRef]

16. Lin, L.; Adey, C. Presentation of Hemiplegic Migraine-Hemiplegia and Hemi-Sensory Loss following General Anaesthesia. Anaesth. Intensiv. Care 2007, 35, 418-422. [CrossRef]

17. Pianezza, A.; Barthélémy, R.; Minville, V.; Martin, F.; Faggianelli, M. Migraine with Atypical Aura in the Recovery Room: A Sometimes Complicated Diagnosis! Anesth. Analg. 2008, 106, 1844-1846. [CrossRef]

18. Willson, J.; Kapur, S. Apnoeic spells following general anaesthesia in a patient with familial hemiplegic migraine. Anaesthesia 2007, 62, 956-958. [CrossRef] [PubMed]

19. Levo, H.; Blomstedt, G.; Hirvonen, T.; Pyykko, I. Causes of persistent postoperative headache after surgery for vestibular schwannoma. Clin. Otolaryngol. 2001, 26, 401-406. [CrossRef]

20. Munkvold, B.K.R.; Sagberg, L.M.; Jakola, A.S.; Solheim, O. Preoperative and Postoperative Headache in Patients with Intracranial Tumors. World Neurosurg. 2018, 115, e322-e330. [CrossRef] [PubMed]

21. Venkatraghavan, L.; Li, L.; Bailey, T.; Manninen, P.H.; Tymianski, M. Sumatriptan improves postoperative quality of recovery and reduces postcraniotomy headache after cranial nerve decompression. Br. J. Anaesth. 2016, 117, 73-79. [CrossRef]

22. Dai, Y.-X.; Tai, Y.-H.; Chang, Y.-T.; Chen, T.-J.; Chen, M.-H. Bidirectional Association between Psoriasis and Atopic Dermatitis: A Nationwide Population-Based Cohort Study. Dermatology 2021, 237, 521-527. [CrossRef]

23. Chen, M.-H.; Dai, Y.-X.; Tai, Y.-H.; Chang, Y.-T.; Chen, T.-J. Increased risk of alopecia areata among patients with endometriosis: A longitudinal study in Taiwan. Dermatol. Sin. 2021, 39, 41. [CrossRef]

24. Chung, Y.-H.; Kuo, H.-C.; Liu, H.-Y.; Wu, M.-Y.; Chang, W.-J.; Chen, J.-T.; Cherng, Y.-G.; Chen, T.-J.; Dai, Y.-X.; Wu, H.-L.; et al Association between Dental Scaling and Reduced Risk of End-Stage Renal Disease: A Nationwide Matched Cohort Study. Int. J. Environ. Res. Public Health 2021, 18, 8910. [CrossRef]

25. Wu, Y.-M.; Kuo, H.-C.; Li, C.-C.; Wu, H.-L.; Chen, J.-T.; Cherng, Y.-G.; Chen, T.-J.; Dai, Y.-X.; Liu, H.-Y.; Tai, Y.-H. Preexisting Dementia Is Associated with Increased Risks of Mortality and Morbidity Following Major Surgery: A Nationwide Propensity Score Matching Study. Int. J. Environ. Res. Public Health 2020, 17, 8431. [CrossRef]

26. National Health Insurance Research Database. Data Subsets. Available online: https://nhird.nhri.org.tw/en/Data_Subsets.html (accessed on 15 November 2021).

27. Mayans, L.; Walling, A. Acute Migraine Headache: Treatment Strategies. Am. Fam. Physician 2018, 97, 243-251. [PubMed]

28. May, A.; Schulte, A.M.L.H. Chronic migraine: Risk factors, mechanisms and treatment. Nat. Rev. Neurol. 2016, 12, 455-464. [CrossRef]

29. Tai, Y.-H.; Wu, H.-L.; Mandell, M.S.; Lin, S.-P.; Tsou, M.-Y.; Chang, K.-Y. The association of non-small cell lung cancer recurrence with allogenic blood transfusion after surgical resection: A propensity score analysis of 1,803 patients. Eur. J. Cancer 2020, 140, 45-54. [CrossRef]

30. Tai, Y.H.; Wu, H.L.; Mandell, M.S.; Tsou, M.Y.; Chang, K.Y. The association of allogeneic blood transfusion and the recurrence of hepatic cancer after surgical resection. Anaesthesia 2020, 75, 464-471. [CrossRef] [PubMed]

31. Wu, H.-L.; Tai, Y.-H.; Lin, S.-P.; Chan, M.-Y.; Chen, H.-H.; Chang, K.-Y. The Impact of Blood Transfusion on Recurrence and Mortality Following Colorectal Cancer Resection: A Propensity Score Analysis of 4030 Patients. Sci. Rep. 2018, 8, 13345. [CrossRef]

32. Dhakal, L.P.; Harriott, A.; Capobianco, D.J.; Freeman, W.D. Headache and Its Approach in Today's NeuroIntensive Care Unit. Neurocrit. Care 2016, 25, 320-334. [CrossRef]

33. Singhal, A.B.; Topcuoglu, M.A. Glucocorticoid-associated worsening in reversible cerebral vasoconstriction syndrome. Neurology 2017, 88, 228-236. [CrossRef]

34. Koch, K.U.; Mikkelsen, I.K.; Aanerud, J.; Espelund, U.S.; Tietze, A.; Oettingen, G.V.; Juul, N.; Nikolajsen, L.; Østergaard, L.; Rasmussen, M. Ephedrine versus Phenylephrine Effect on Cerebral Blood Flow and Oxygen Consumption in Anesthetized Brain Tumor Patients. Anesthesiology 2020, 133, 304-317. [CrossRef]

35. Bath, P.M. Theophylline, aminophylline, caffeine and analogues for acute ischaemic stroke. Cochrane Database Syst. Rev. 2004, 2004, CD000211. [CrossRef] 
36. Austin, P.C. Balance diagnostics for comparing the distribution of baseline covariates between treatment groups in propensityscore matched samples. Stat. Med. 2009, 28, 3083-3107. [CrossRef] [PubMed]

37. Lyngberg, A.C.; Rasmussen, B.K.; Jørgensen, T.; Jensen, R. Incidence of Primary Headache: A Danish Epidemiologic Follow-up Study. Am. J. Epidemiol. 2005, 161, 1066-1073. [CrossRef]

38. Pellegrino, A.B.W.; E Davis-Martin, R.; Houle, T.; Turner, D.P.; Smitherman, T. Perceived triggers of primary headache disorders: A meta-analysis. Cephalalgia 2017, 38, 1188-1198. [CrossRef]

39. Jette, N.; Patten, S.; Williams, J.; Becker, W.; Wiebe, S. Comorbidity of Migraine and Psychiatric Disorders-A National PopulationBased Study. Headache J. Head Face Pain 2008, 48, 501-516. [CrossRef] [PubMed]

40. Breslau, N.; Lipton, R.B.; Stewart, W.F.; Schultz, L.R.; Welch, K.M. Comorbidity of migraine and depression: Investigating potential etiology and prognosis. Neurology 2003, 60, 1308-1312. [CrossRef]

41. Silberstein, S.D.; Lee, L.; Gandhi, K.; Ma, T.F.; Bell, J.; Cohen, J.M. Health care Resource Utilization and Migraine Disability Along the Migraine Continuum Among Patients Treated for Migraine. Headache J. Head Face Pain 2018, 58, 1579-1592. [CrossRef]

42. Sumelahti, M.-L.; Sumanen, M.; Sumanen, M.S.; Tuominen, S.; Vikkula, J.; Honkala, S.M.; Rosqvist, S.; Korolainen, M.A. My Migraine Voice survey: Disease impact on healthcare resource utilization, personal and working life in Finland. J. Headache Pain 2020, 21, 118. [CrossRef]

43. Woldeamanuel, Y.W.; Rapoport, A.M.; Cowan, R.P. What Is the Evidence for the Use of Corticosteroids in Migraine? Curr. Pain Headache Rep. 2014, 18, 464. [CrossRef]

44. Orr, S.; Friedman, B.W.; Christie, S.; Minen, M.T.; Bamford, C.; Kelley, N.E.; Tepper, D. Management of Adults With Acute Migraine in the Emergency Department: The American Headache Society Evidence Assessment of Parenteral Pharmacotherapies. Headache J. Head Face Pain 2016, 56, 911-940. [CrossRef] [PubMed]

45. Kelley, N.E.; Tepper, D.E. Rescue Therapy for Acute Migraine, Part 3: Opioids, NSAIDs, Steroids, and Post-Discharge Medications. Headache J. Head Face Pain 2012, 52, 467-482. [CrossRef] [PubMed]

46. Sprenger, T.; Viana, M.; Tassorelli, C. Current Prophylactic Medications for Migraine and Their Potential Mechanisms of Action Neurotherapeutics 2018, 15, 313-323. [CrossRef] [PubMed]

47. Sørensen, H.; Rasmussen, P.; Sato, K.; Persson, S.; Olesen, N.D.; Nielsen, H.B.; Olsen, N.V.; Ogoh, S.; Secher, N.H. External carotid artery flow maintains near infrared spectroscopy-determined frontal lobe oxygenation during ephedrine administration. Br. $J$. Anaesth. 2014, 113, 452-458. [CrossRef]

48. Devereux, G.; Cotton, S.; Fielding, S.; McMeekin, N.; Barnes, P.J.; Briggs, A.; Burns, G.; Chaudhuri, R.; Chrystyn, H.; Davies, L.; et al. Effect of Theophylline as Adjunct to Inhaled Corticosteroids on Exacerbations in Patients With COPD: A Randomized Clinical Trial. JAMA 2018, 320, 1548-1559. [CrossRef] [PubMed]

49. Shannon, M. Life-Threatening Events after Theophylline Overdose. Arch. Intern. Med. 1999, 159, 989-994. [CrossRef] [PubMed]

50. Sayyah, M.; Saki-Malehi, A.; Javanmardi, F.; Forouzan, A.; Shirbandi, K.; Rahim, F. Which came first, the risk of migraine or the risk of asthma? A systematic review. Neurol. Neurochir. Polska 2018, 52, 562-569. [CrossRef] [PubMed]

51. Martin, V.T.; Fanning, K.M.; Serrano, D.; Buse, D.C.; Reed, M.L.; Lipton, R.B. Asthma is a risk factor for new onset chronic migraine: Results from the American migraine prevalence and prevention study. Headache J. Head Face Pain 2015, 56, 118-131. [CrossRef]

52. Maniaci, A.; Merlino, F.; Cocuzza, S.; Iannella, G.; Vicini, C.; Cammaroto, G.; Lechien, J.R.; Calvo-Henriquez, C.; La Mantia, I. Endoscopic surgical treatment for rhinogenic contact point headache: Systematic review and meta-analysis. Eur. Arch. Oto-Rhino-Laryngol. 2021, 278, 1743-1753. [CrossRef]

53. Coeytaux, R.R.; Befus, D. Role of Acupuncture in the Treatment or Prevention of Migraine, Tension-Type Headache, or Chronic Headache Disorders. Headache J. Head Face Pain 2016, 56, 1238-1240. [CrossRef] 